\title{
Primary Central Nervous System Lymphoma (PCNSL): Analysis of Treatment by Gamma Knife Radiosurgery and Chemotherapy in a Prospective, Observational Study
}

\author{
Andres M. Alvarez-Pinzon ${ }^{1}$, Aizik L. Wolf ${ }^{2}$, Heather Swedberg ${ }^{3}$, Sammie R. Coy ${ }^{1}$, Jose E. \\ Valerio $^{1}$ \\ 1. Miami Neuroscience Center, Larkin Community Hospital 2. Miami Neuroscience Center, Larkin \\ Community Hospital, MIami, USA 3. Larkin Community Hospital, St. George's University School of \\ Medicine
}

$\square$ Corresponding author: Heather Swedberg, hswedber@sgu.edu

Disclosures can be found in Additional Information at the end of the article

\section{Abstract}

Background:

Primary central nervous system lymphoma (PCNSL) is a rare cancer accounting for less than 3\% of primary brain and central nervous system (CNS) tumors. Tissues involved include the brain parenchyma, leptomeninges, eyes, and spinal cord. High-dose methotrexate (MTX) is the gold standard for newly diagnosed PCNSL. However, Gamma Knife radiosurgery (GKRS) may be efficacious as a co-adjuvant treatment. The purpose of this prospective observational cohort study is to determine the effectiveness of MTX in combination with GKRS in the treatment of PCNSL.

Methods:

This is a prospective, observational cohort study evaluating the treatment of histologically confirmed PCNSL with MTX as a single agent in a dose of $8 \mathrm{~g} / \mathrm{m} 2$ (control) and treatment with MTX, plus GKRS. Strict inclusion and exclusion criteria were employed. Primary outcomes were measured by survival rate. Secondary outcomes were assessed by the tumor's responsiveness to treatment and reduction in size as noted on imaging.

Results:

Between January 2007 and January 2012, 128 charts were evaluated. Included in this evaluation were 73 chemotherapy (control) and 55 chemotherapy, plus GKRS, patients (variable). The follow-up period was 24 to 49 months (mean: 36.9 months). There were no statistically significant differences in patient demographics or histology diagnosis. Patients were treated with GK doses ranging from $11 \mathrm{~Gy}$ to $16 \mathrm{~Gy}$ (median: $11 \mathrm{~Gy}$ ). The median survival rate from initial diagnosis was 26.8 months in the chemotherapy group and 47.6 in the chemotherapy, plus GKRS, group (p-value: 0.0034). All lesions showed a complete response after GKRS when evaluated using magnetic resonance imaging after three to eight weeks (mean range: 6.3 weeks).

Conclusions:

\section{How to cite this article}

Alvarez-Pinzon A M, Wolf A L, Swedberg H, et al. (July 18, 2016) Primary Central Nervous System Lymphoma (PCNSL): Analysis of Treatment by Gamma Knife Radiosurgery and Chemotherapy in a Prospective, Observational Study . Cureus 8(7): e697. DOI 10.7759/cureus.697 
The use of GKRS is non-invasive, safe, and shows rapid success, improving the prognosis of the patient. This noninvasive treatment modality should be considered as an option for patients with PCNSL. In our study, GKRS as a co-adjuvant therapy to high-dose methotrexate was statistically significant for greater tumor control, enhanced overall survival period, and a lesser number of complications.

Categories: Neurology, Neurosurgery, Oncology

Keywords: primary cns lymphoma, radiosurgery, gamma knife, chemotherapy, brain tumor, methotrexate, lymphoma, pcnsl, gkrs

\section{Introduction}

Primary central nervous system lymphoma (PCNSL) is a rare cancer accounting for less than 3\% of primary brain and central nervous system (CNS) tumors [1]. PCNSL routinely presents as a single or multifocal lesion capable of infiltrating the cortex, with extension into the white and/or gray matter. Areas of necrosis may be found, especially in the immunocompromised. The primary CNS extranodal high-grade malignant cells are often large or immunoblastic cells of B-cell non-Hodgkin's, originating from the brain parenchyma, spinal cord, leptomeninges, or eyes [2]. PCNSL is typically limited to the CNS, often as intra-axial nodules with diffuse extension throughout the meningeal, periventricular, and perivascular spaces.

The prognosis of untreated PCNSL remains grim with low median survival time; therefore, the most effective treatment should be initiated as soon as possible [3]. In comparison to other brain tumors, resection is not indicated for the treatment of newly diagnosed PCNSL; management with radiation and/or chemotherapy is recommended [4-5]. Gamma Knife radiosurgery (GKRS), also called stereotactic radiosurgery, is a non-invasive treatment that utilizes beams of gamma radiation to target a specific lesion. While GKRS has not yet been used universally in the treatment of the PCNSL, it is a relatively safe alternative, yielding rapid and successful results, and ultimately improving the prognosis and quality of life of the patient [6]. The role of radiation therapy for initial management of PCNSL, however, remains controversial. Clinical trials should attempt to improve the therapeutic index of this modality.

A major challenge in the management of patients with PCNSL remains the delivery of aggressive chemotherapeutic treatment with preservation of neurocognitive function. Routes of chemotherapy administration include intravenous, intraocular, intraventricular, or intraarterial. Multiple trials have outlined different methotrexate-based chemotherapy regimens and are utilizing local techniques to improve drug delivery. A study of the blood-brain barrier (BBB) permeability in a rat brain tumor model established a large heterogeneity of microvascular leakage; the vasculature within and around the brain tumors has a wide range of permeability, from typical capillaries with no (BBB) leakage to tumor vasculature allowing free entry of large molecules [7]. High-dose MTX $\left(>1 \mathrm{~g} / \mathrm{m}^{2}\right)$ has been shown to be an independent factor correlating with survival. Thus, MTX is administered in high doses, up to $8 \mathrm{~g} / \mathrm{m}^{2}$, in order to achieve therapeutic drug concentrations in the tumor and surrounding brain parenchyma [7]. Intravenous (IV) doses less than $0.5 \mathrm{~g} / \mathrm{m}^{2}$, similar to what has been used in the treatment of malignancies outside of the brain, reach CNS concentrations generally thought to be noncytotoxic [7].

The purpose of this prospective observational cohort study is to determine the effectiveness of chemotherapy using MTX in doses of $8 \mathrm{~g} / \mathrm{m}^{2}$ in combination with GKRS in the treatment of PCNSL. 


\section{Cureus}

\section{Materials And Methods}

This is a prospective observational cohort study evaluating the effect and survival rate of patients with a histological diagnosis of PCNSL treated with MTX alone (control) and MTX, plus GKRS (variable). Charts were reviewed between January 2007 and January 2012. The study received approval from the Larkin Community Hospital Institutional Review Board (approval \#LCH - 022015). The IRB waived the informed consent process. A single experienced, blinded post-doctoral fellow used Research Electronic Data Capture (REDCap) to collect data from eligible patients. Eligible patients underwent GKRS and/or a chemotherapy protocol using MTX at the same institution. All patients included in the study were diagnosed with PCNSL by biopsy and pathohistology report of the brain lesion observed on MRI. Strict inclusion and exclusion criteria were employed, as outlined in Table 1.

\section{INCLUSION AND EXCLUSION CRITERIA}

Inclusion Criteria

- Patients with a diagnosis of PCNSL and treated by Methotrexate

- Primary CNS lymphoma diagnosed by neurohistology pathologist

\section{Exclusion Criteria}

- AIDS diagnosis at time of treatment and diffuse CNS lymphomas

- Patients with addiction to illegal drugs, solvents, or alcohol who are currently using or previously attempted or failed a treatment program

- Patients with bacteremia, systemic infection, or infection

- Patients pregnant or nursing

- History of previous brain surgery or brain tumor

- History of systemic disease

\section{TABLE 1: Inclusion and Exclusion Criteria}

Primary inclusion criteria were subjects who received MTX therapy as a single agent in a standard protocol dosage of $8 \mathrm{~g} / \mathrm{m}^{2}$. The presence and size of the tumor, as well as its localization, were confirmed using a brain MRI protocol. The maximal brain tumor was measured at $1 \mathrm{~cm}$ and $2 \mathrm{~cm}$ proximal to the most distal aspect of its midline. Primary outcomes were measured by survival rate. Secondary outcomes were assessed by the tumor's responsiveness to treatment and reduction in size as noted on MRI and/or CT scan.

\section{Statistical analysis}

A priori sample size calculation was performed on the basis of a prior study, which evaluated the survival rate in a sample size of 40 patients [8]. The study implied a sample size of at least 31 subjects per group is necessary to detect the minimal clinically important difference in the mortality rate of 1.5 standard deviations $(\sigma=1.5, \alpha=0.05, \beta=0.20)$. Additionally, a general estimate for detecting a one-unit change on an ordinal scale of $136(\sigma=1.0, \alpha=0.05, \beta=0.20)$ resulted in the same number. The Wilcoxon Rank-Sum Test was used for comparison of continuous data between the groups. Differences between means were analyzed using twosided T-tests. Comparison of categorical data was performed using Pearson's Chi-square test or Fisher's exact test as indicated. Ordinal ranking scores were compared using the Mantel- 


\section{Cureus}

Haenszel test.

\section{Results}

One hundred twenty-eight charts were evaluated between January 2007 and January 2012. Included were 73 chemotherapy and 55 chemotherapy, plus GKRS, patients. The follow-up period was 24 to 49 months (mean 36.9 months). There were no statistically significant differences in patient demographics or histology diagnosis (Table 2).

\section{Demographic and Perioperative Data}

\begin{tabular}{|c|c|c|c|}
\hline Patient Parameters & Control & GK Group & $P$ value \\
\hline Number of Patients & 73 & 55 & NA \\
\hline Age (years) & {$[58.1 \pm 5.3]$} & {$[56.9 \pm 3.3]$} & 0.685 \\
\hline \multicolumn{4}{|l|}{ Sex } \\
\hline Male & $33(70 \%)$ & $26(56 \%)$ & 0.51 \\
\hline Female & $40(30 \%)$ & $19(44 \%)$ & \\
\hline Body-mass index $\left(\mathrm{kg} / \mathrm{m}^{2}\right)$ & {$[24.1 \pm 2.6]$} & {$[22.2 \pm 3.1]$} & 0.39 \\
\hline \multicolumn{4}{|l|}{ Preop Diagnosis } \\
\hline Tobacco use & 16 & 22 & 0.25 \\
\hline Pre-albumin (mg/dL) & {$[18.1 \pm 1.3]$} & {$[19.1 \pm 0.9]$} & 0.347 \\
\hline Albumin (g/dL) & {$[3.4 \pm 0.4]$} & {$[3.2 \pm 0.4]$} & $0.89 \dagger$ \\
\hline Absolute Lymphocyte Count (cells/ $\mu \mathrm{L}$ ) & {$[1745 \pm 468]$} & {$[1798 \pm 401]$} & 0.20 \\
\hline Median Survival & 26.8 months $(18.3-29.1)$ & 47.6 months $(28.3-49.1)$ & 0.0034 \\
\hline Preop Diagnosis & & & $0.59 \dagger$ \\
\hline
\end{tabular}

\section{TABLE 2: Patient Demographic and Perioperative Data}

Values are given as mean and standard deviation, with or without range in parentheses and 95\% confidence interval in brackets.

† Relationship between the number of positive cases and the total number of assessed patients.

The histologic finding in all patients was the presence of large, diffuse B-cell lymphoma. Patients were treated with GKRS doses ranging from $11 \mathrm{~Gy}$ to $16 \mathrm{~Gy}$ (median: $11 \mathrm{~Gy}$ ) 50\% isodose line (Figure 1). 


\section{Cureus}

\section{Mets / lymphoma}

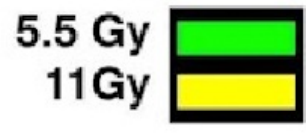

\section{1st Row \\ 2nd Row}
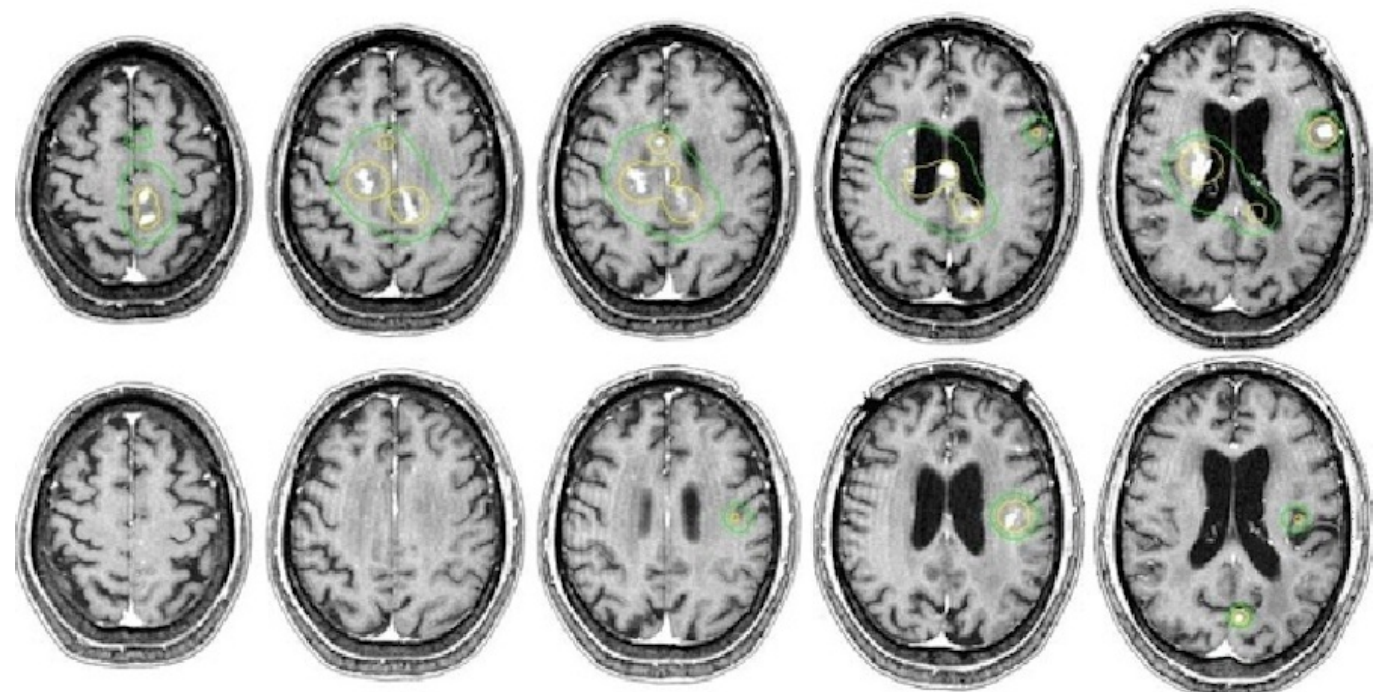

FIGURE 1: Brain MRI before and two months after GKRS treatment

The median survival rate from initial diagnosis was statistically significant $(p=0.0034)$ with 26.8 months in the chemotherapy group and 47.6 months in patients receiving chemotherapy, plus GKRS. The signs and symptoms of the patients remained evidently improved within two to six weeks after GKRS and four to 10 weeks after chemotherapy alone. Lesions treated with GKRS showed a complete response on MRI when evaluated three to eight weeks (mean range: 6.3 weeks) post-therapy.

The clinical data was gathered for brain tumor lesion control, disease control, toxicity, recurrence, and survival as seen in Table 3. 


\section{Cureus}

\section{Brain Tumor-Related Outcomes}

Patient Parameters

Control Group

Gamma Knife

P Value

Tumor Size

Mean Size $\mathrm{cm}^{3}$

3.7

0.18

Median Size $\mathrm{cm}^{3}$

2.9

2.7

Size Range $\mathrm{cm}^{3}$

$1.3-6.9$

$1.5-6.4$

Presence of single or multiple lesions (\%)

Single

Multiple

$92 \%$

$78 \%$

0.28

Tumor location

Frontal

Parietal

Temporal

19

14

0.09

Occipital

7

7

0.58

Cerebellum

6

4

Extracranial metastasis

Yes

No

VP Shunt

Yes

No

Prior cancer

Systemic symptoms

Positive CSF cytology examination

High CSF protein level

Multiple lesions
24

49

11

5

73

51

42
14

0.068

41

0.35

0.24

0.587

0.187

0.14

0.0687

\section{TABLE 3: Brain Tumor-Related Outcomes}


postoperative average of $88 \%$ in the GKRS group. Significant factors contributing to survival greater than 24 months following GKRS were increased marginal dose (odds ratio $=6.5, \mathrm{p}=$ $0.031,95 \%$ CI (1.4-18.57)), increase in maximal dose (odds ratio $=1.54, p=0.012,95 \%$ CI $(1.15$ 2.89 )), and increase in the pretreatment Karnofsky score (odds ratio $=5.13, \mathrm{p}=0.008,95 \% \mathrm{CI}$ (1.55-85.1)). The side effects attributed to GKRS were minimal; none of the patients had a deterioration related to the treatment. No complications were related after the procedure.

\section{Discussion}

The dilemma faced in the treatment of PCNSL is the determination of a superior approach that will offer higher success rates while limiting adverse effects, such as neurotoxicity. While the gold standard therapy for patients with newly diagnosed PCNSL remains high-dose MTX, the use of higher doses of MTX regimens in elderly patients may be associated with a higher risk of systemic toxicity because of the increased prevalence of comorbidities [9]. Combination chemotherapy regimens, such as the methotrexate, cytarabine, thiotepa, and rituximab (MATRix) regimen, are associated with a greater amount of hematologic complications, including anemia, neutropenia, and thrombocytopenia [10]. Historically, there has been a high incidence of severe neurotoxicity in patients receiving whole brain radiotherapy (WBRT), predominantly those older than 65 years of age [2, 11-12]. Moreover, up to 35-50\% of patients present with neurologic deterioration after WBRT, resulting in a five-year mortality rate of $30 \%$ $[2,13]$.

GKRS, in comparison to WBRT, provides targeted radiation to the lesion, perhaps attributing to a lower incidence of neurotoxicity. Of note, not a single patient experienced any mental or memory impairment in our study, which is in partial agreement with an earlier report [6]. Kenai, et al. described a mean recurrence-free period of 24.4 months for their patients [6]. In our observational study, there were 16 patients each with more than seven brain lesions, an association with poor clinical prognosis. Nine patients developed new lesions in other areas of the brain and repeated GKRS was performed. No local recurrence was observed in any patient at a mean of 30.6 months post-GKRS treatment. The median survival rate from initial diagnosis was statistically significant (p-value: 0.0034 ) with 26.8 months in the chemotherapy group and 47.6 months in patients receiving chemotherapy, plus GKRS. Therefore, our findings are in accordance with those of Kenai and seem to be at a higher success rate than those described by others in treating patients with chemotherapy and/or radiotherapy alone.

While there appeared to be a predisposition in the direction of new tumor growth in individuals who did not undergo GKRS treatment, we do not suggest a connection between recurrence and original appearance of multiple or solitary lesions, but current systemic chemotherapy would be essential. Overall, GKRS appears to be a valuable method for the management of primary and relapsed CNSL, since it enables exceptional local control in a short-term period without severe neurotoxicity or complications [14].

\section{Conclusions}

GKRS is a minimally invasive procedure that allows for the immediate use of systemic chemotherapy and can be an ideal co-adjuvant treatment option in patients with PCNSL. The combination allows for greater tumor control, enhanced overall survival period, and a lesser number of complications.

\section{Additional Information Disclosures}

Human subjects: Consent was obtained by all participants in this study. Larkin Community Hospital issued approval LCH - 022015. The study is part of the brain pathology registry. The 
research involves no more than minimal risk to the subjects. Whenever appropriate the subjects will be provided with additional pertinent information after participation. The IRB waived the informed consent process. Animal subjects: All authors have confirmed that this study did not involve animal subjects or tissue. Conflicts of interest: In compliance with the ICMJE uniform disclosure form, all authors declare the following: Payment/services info: All authors have declared that no financial support was received from any organization for the submitted work. Financial relationships: All authors have declared that they have no financial relationships at present or within the previous three years with any organizations that might have an interest in the submitted work. Other relationships: All authors have declared that there are no other relationships or activities that could appear to have influenced the submitted work.

\section{References}

1. Ostrom QT, Gittleman H, Farah P, Ondracek A, Chen Y, Wolinsky Y, Stroup NE, Kruchko C, Barnholtz-Sloan JS: CBTRUS statistical report: Primary brain and central nervous system tumors diagnosed in the United States in 2006-2010. Neuro Oncol. 2013, 15:ii1-56. 10.1093/neuonc/not151

2. Wang CC, Carnevale J, Rubenstein JL: Progress in central nervous system lymphomas . Br J Haematol. 2014, 166:311-25. 10.1111/bjh.12938

3. Liu HL, Liu T, Ma C, Zhao Y, Zhou Y: Clinical characteristics and prognosis of 20 cases of primary central nervous system lymphoma (Article in Chinese) . J Experimental Hematol. 2015, 23:111-18.

4. Weller M, Martus P, Roth P, Thiel E, Korfel A; German PCNSL Study Group: Surgery for primary CNS lymphoma? Challenging a paradigm. Neuro Oncol. 2012, 14:1481-84. 10.1093/neuonc/nos159

5. Beiko J, Suki D, Hess KR, Fox BD, Cheung V, Cabral M, Shonka N, Gilbert MR, Sawaya R, Prabhu SS, Weinberg J, Lang FF, Aldape KD, Sulman EP, Rao G, McCutcheon IE, Cahill DP: IDH1 mutant malignant astrocytomas are more amenable to surgical resection and have a survival benefit associated with maximal surgical resection. Neuro Oncol. 2014, 16:81-91. 10.1093/neuonc/not159

6. Kenai H, Yamashita M, Nakamura T, Asano T, Momii Y, Nagatomi H: Gamma Knife surgery for primary central nervous system lymphoma: usefulness as palliative local tumor control. J Neurosurg. 2006, 105:133-38.

7. Jahnke K, Doolittle ND, Muldoon LL, Neuwelt EA: Implications of the blood-brain barrier in primary central nervous system lymphoma. Neurosurg Focus. 2006, 21:E11. 10.3171/foc. 2006.21.5.12

8. Mahadevan A, Rao CR, Shanmugham M, Shankar SK: Primary central nervous system diffuse large B-cell lymphoma in the immunocompetent: Immunophenotypic subtypes and EpsteinBarr virus association. J Neurosci Rural Pract. 2015, 6:8-14. 10.4103/0976-3147.143171

9. Zhu JJ, Gerstner ER, Engler DA, Mrugala MM, Nugent W, Nierenberg K, Hochberg FH, Betensky RA, Batchelor TT: High-dose methotrexate for elderly patients with primary CNS lymphoma . Neuro Oncol. 2009, 11:211-15. 10.1215/15228517-2008-067

10. Ferreri A, Cwynarski K, Pulczynski E, Ponzoni M, Deckert M, Politi LS, Torri V, Fox CP, Rosée PL, Schorb E, Ambrosetti A, Roth A, Hemmaway C, Ferrari A, Linton KM, Rudà R, Binder M, Pukrop T, Balzarotti M, Fabbri A, Johnson P, Gørløv JS, Hess G, Panse J, Pisani F, Tucci A, Stilgenbauer S, Hertenstein B, Keller U, Krause SW, Levis A, Schmoll HJ, Cavalli F, Finke J, Reni M, Zucca E, Illerhaus G; International Extranodal Lymphoma Study Group (IELSG): Chemoimmunotherapy with methotrexate, cytarabine, thiotepa, and rituximab (MATRix regimen) in patients with primary CNS lymphoma: results of the first randomisation of the International Extranodal Lymphoma Study Group-32 (IELSG32) phase 2 trial. Lancet Haematol. 2016, 3:e217-27. 10.1016/S2352-3026(16)00036-3

11. Thiel E, Korfel A, Martus P, Kanz L, Griesinger F, Rauch M, Röth A, Hertenstein B, von Toll T, Hundsberger T, Mergenthaler HG, Leithäuser M, Birnbaum T, Fischer L, Jahnke K, Herrlinger U, Plasswilm L, Nägele T, Pietsch T, Bamberg M, Weller M: High-dose methotrexate with or without whole brain radiotherapy for primary CNS lymphoma (G-PCNSL-SG-1): a phase 3, randomised, non-inferiority trial. Lancet Oncol. 2010, 11:1036-47. 10.1016/S1470-

2045(10)70229-1 


\section{Cureus}

12. Rubenstein JL, Hsi ED, Johnson JL, Jung SH, Nakashima MO, Grant B, Cheson BD, Kaplan LD: Intensive chemotherapy and immunotherapy in patients with newly diagnosed primary CNS lymphoma: CALGB 50202 (Alliance 50202). J Clin Oncol. 2013, 31:3061-68.

10.1200/JCO.2012.46.9957

13. Perini GF, Campregher PV, Santos FP, Hamerschlak N: Primary central nervous system lymphoma: what a neurologist/neurosurgeon should know?. Arq Neuropsiquiatr. 2013, 71:254-57. 10.1590/0004-282X20130011

14. Matsumoto Y, Horiike S, Fujimoto Y, Shimizu D, Kudo-Nakata Y, Kimura S, Sato M, Nomura K, Kaneko H, Kobayashi Y, Shimazaki C, Taniwaki M: Effectiveness and limitation of gamma knife radiosurgery for relapsed central nervous system lymphoma: a retrospective analysis in one institution. Int J Hematol. 2007, 85:333-37. 10.1532/IJH97.06205 